\title{
Effect Of Non-Darcian Flow On Natural Convection Inside Rectangular Cavity With Saturated Porous Media Using Vertical Partition
}

\author{
Abbas S. Hussain \\ Ziad M. Al-Makhyoul \\ Assistant Lecturer \\ Ramiz I. Al-Aboshi \\ Lecturer
}

Department of Mechanical Engineering -University of Mosul - Iraq

\begin{abstract}
This numerical study documents the effect of an adiabatic internal flow partition on natural convection heat transfer in a two dimensional enclosure filled with saturated porous media whereas the partition attached vertically on the lower insolated wall. The study concluded the effect of partition using different lengths $\left(\mathrm{L}_{\mathrm{P}}=0.3,0.5\right.$ and 0.7$)$, locations $\left(\mathrm{X}_{\mathrm{P}}=0.3,0.5\right.$ and 0.7$)$ and different inertia effect $\left(\mathrm{Fs} / \mathrm{Pr}=10^{-2}, 10^{-3}\right.$ and $\left.10^{-4}\right)$ on heat transfer with aspect ratio $(A=1.0)$. A continuity, energy and momentum equations are analyzed and solved using finite difference with Gauss-siedel iterative method. From results, it's clear that the partition caused a deflection on stream lines causing a disturbance in the flow patterns. This affected the heat transfer rate represented by Nusselt number. Also, for a wide range of governing parameter, it may be concluded that the partition reduces the heat transfer rate.
\end{abstract}

Key Words: Heat transfer, Porous media, Natural convection, partition, Enclosure

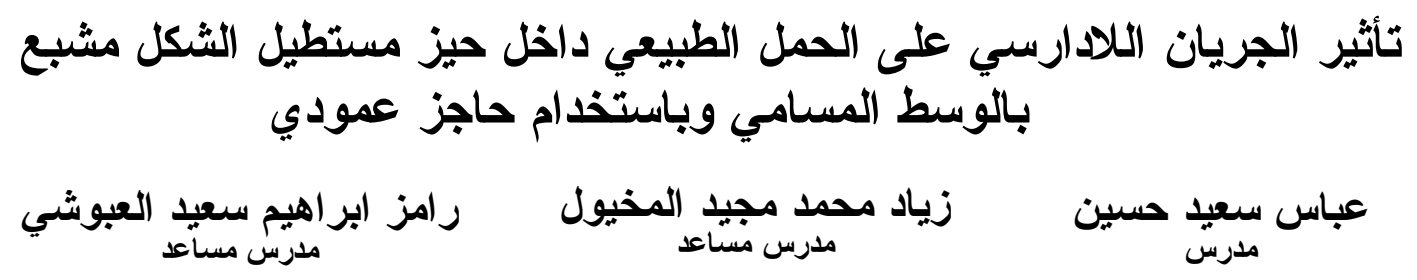

جامعة الموصل- قسم الهندسة الميكانيكية ـكلية الهندسة

\section{الخلاصة}

توثق هذه الدراسة العددية تأثير الحاجز الداخلي الاديباتي على انتقال الحرارة بالحمل الطبيعي داخل حيز ثنائي

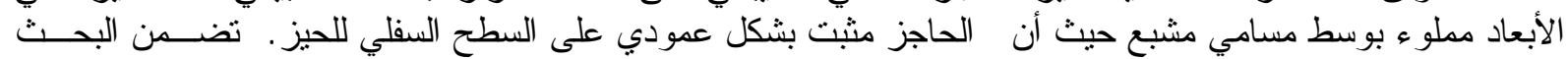

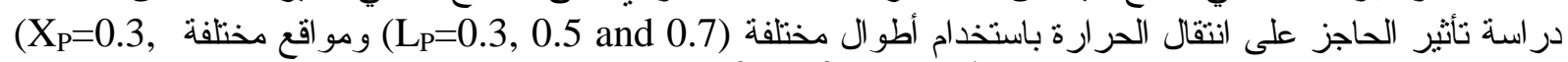

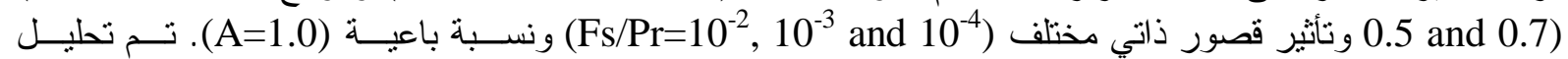

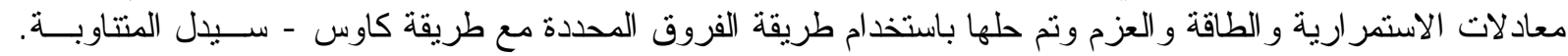

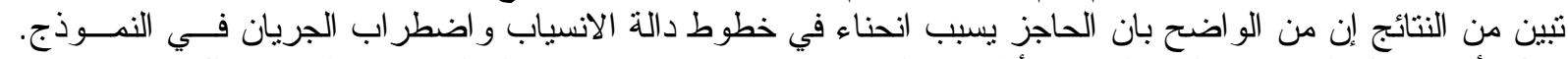

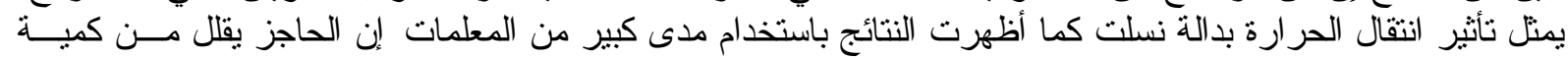

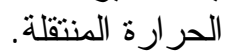




\begin{tabular}{llll} 
Al-Rafidain Engineering & Vol.17 & No.3 & June 2009 \\
\hline
\end{tabular}

Nomenclature

$\begin{array}{lll}\mathrm{A} & \text { Aspect ratio } & \mathrm{H} / \mathrm{L} \\ \mathrm{Da} & \text { Darcy number } & \mathrm{K} / \mathrm{L}^{2} \\ \mathrm{Fs} & \text { Forchhimer number } & \mathrm{b} / \mathrm{L} \\ \mathrm{g} & \text { Gravititional acceleration } & \mathrm{m} / \mathrm{s}^{2} \\ \mathrm{Gr} & \text { Grashof number } & \mathrm{g} \beta \mathrm{L}^{3} \Delta \mathrm{T} / \mathrm{v}^{2} \\ \mathrm{~h} & \text { Heat transfer coefficient } & \mathrm{W} / \mathrm{m}^{2} \cdot \mathrm{K} \\ \mathrm{L}_{\mathrm{p}} & \text { Height of partition } & \mathrm{m} \\ \mathrm{H} & \text { Enclosure height } & \mathrm{m} \\ \mathrm{k} & \text { Thermal conductivity } & \mathrm{W} / \mathrm{m} \cdot \mathrm{K} \\ \mathrm{k}_{\mathrm{e}} & \text { Effective thermal conductivity } & \mathrm{W} / \mathrm{m} \cdot \mathrm{K} \\ \mathrm{L} & \text { Enclosure width } & \mathrm{m} \\ \mathrm{Nu} & \text { Nusselt number } & \mathrm{hL} / \mathrm{k}_{\mathrm{e}} \\ \mathrm{Pr} & \text { Prandetl number } & \mathrm{v} / \alpha \\ \mathrm{Q} & \text { Heat transfer from side } & \mathrm{W} \\ \mathrm{Re} & \text { Reynolds number } & \mathrm{vL} / \mu \\ \mathrm{Ra} & \text { Rayleigh number } & \mathrm{g} \beta \mathrm{L}^{3} \Delta \mathrm{T} / \alpha \mathrm{v} \\ \mathrm{Ra} & \text { Modify Rayleigh number } & \mathrm{g} \beta \mathrm{LK} \Delta \mathrm{T} / \alpha \mathrm{v} \\ \mathrm{T} & \text { Temperature } & \mathrm{K} \\ \Delta \mathrm{T} & \text { Temperature difference }\left(\mathrm{T}_{\mathrm{h}}-\mathrm{T}_{\mathrm{c}}\right) & \mathrm{K} \\ \mathrm{t}_{\mathrm{p}} & \text { Partition thickness } & \mathrm{m} \\ \mathrm{u}, \mathrm{v} & \text { Velocity in x and y direction } & \mathrm{m} / \mathrm{s} \\ \hat{\mathrm{u}}, \hat{\mathrm{v}} & \text { Dimensionless velocity in } \mathrm{x} \text { and y direction } & \\ \mathrm{V} & \text { Velocity vector } & \mathrm{m} / \mathrm{s} \\ |\mathrm{V}| & \text { Absolute velocity vector } & \mathrm{m} / \mathrm{s} \\ \mathrm{X}_{\mathrm{p}} & \text { Position of partition from hot wall } & \mathrm{m} \\ \mathrm{x}, \mathrm{y} & \text { Cartesian coordinate } & \mathrm{m} \\ \hat{\mathrm{x}} & \text { Dimensionless distance in x-direction } & \mathrm{x} / \mathrm{L} \\ \hat{\mathrm{y}} & \text { Dimensionless distance in y-direction } & \mathrm{y} / \mathrm{L} \\ & & \end{array}$

\section{Greek symbols}

$\alpha$
$\beta$
$\theta$
$\mu$
$v$
$\rho$
$\phi$
$\psi$

Thermal diffusivity

Coefficient of thermal expansion

$\mathrm{m}^{2} / \mathrm{s}$

Dimensionless temperature

$\mathrm{K}^{-1}$

Dynamic viscosity

Kinematic viscosity

Density

Depended variable

Stream function

$\left(\mathrm{T}-\mathrm{T}_{\mathrm{c}}\right) / \Delta \mathrm{T}$

$\mathrm{kg} / \mathrm{m} . \mathrm{s}$

$\mathrm{m}^{2} / \mathrm{s}$

$\mathrm{kg} / \mathrm{m}^{3}$

\section{Subscripts}

$\begin{array}{ll}\mathrm{c} & \text { Cold } \\ \mathrm{h} & \text { Hot } \\ \max & \text { Maximum }\end{array}$




\section{Hussain : Effect Of Non-Darcian Flow On Natural Convection Inside Rectangular}

\section{Introduction:}

The analysis of natural convection heat transfer in porous medium has been an important subject due to its increasing applications in many industrial and geophysical problems, particularly in oil exploration and in the petroleum chemical and nuclear industries. The porous media can be naturally formed (e.g., rocks, sand beds, sponges, woods) or fabricated (e.g., catalytic pellets, wicks, insulation).

Chan et all [1] used numerical method to solve the field equation for heat transfer in a porous medium filled with gas and bounded by plane rectangular surfaces at different temperatures. The results had been presented in terms of theoretical streamlines and isotherms. The relative increase in heat transfer rate corresponding to natural convection is obtained as a function of three dimensionless parameter: Darcy number (Da), the Rayleigh number (Ra) and the geometric aspect ratio (A). Possible correlation using the lumped parameter Da Ra was proposed for a value grater than about 40.

Tong and Subramanian [2] performed a boundary layer analysis for natural convection in a vertical porous enclosure using the Brikman model to derive the boundary layer equation. The flow field was found to be characterized by Rayleigh number, Darcy number and aspect ratio. They concluded that Brikman model offered the best agreement with experimental results.

Prasad and Tuntomo [3] examined inertia effects on buoyancy driven flow and heat transfers in a vertical porous cavity using the Forchheimer- extended Darcy equation of motion for flow through porous media. The dimensional analysis indicates that the inertia added a new dimensionless parameter Fs (Forchheimer number) to the Rayleigh number and Prandtl number Pr. Finite difference numerical results for a wide range of parameter indicated that the effect of Prandtl number and aspect ratio remains unchanged with an enhancement in the inertia effects. An inertia parameter Fs/Pr* was suggested where the velocities decrease when it increases.

Beckermann et al [4] carried out a numerical and experimental study to analyze steady state natural convection fluid flow and heat transfer in a vertical rectangular cavity partially filled with a vertical layer of fluid saturated porous medium. They used BrinkmanForchheimer extended Darcy equation. They concluded that the amount of fluid penetrating from the fluid region in to the porous layer depends strongly on the Darcy number and the Rayleigh number. For a low product of $\mathrm{Ra}$ and $\mathrm{Da}$, the flow took place primarily in the fluid layer, and heat transfer in the porous layer is by conduction only.

Hussain [5] studied the effect of an adiabatic internal flow partition on natural convection heat transfer. It was in two-dimensional enclosures filled with saturated porous media. Deferentially heated on the sides with insulated horizontal walls, the partition is fixed horizontally on the hot wall. The partition has different lengths and positions. He showed that the partition causes convection suppression and heat transfer reduction compared with no partition conditions and the increase in inertia parameter from $10^{-4}$ to $10^{-2}$ causes a reduction in Nusselt number in ranges of $25-35 \%$ for $(A=1.0), 10-20 \%$ for $(A=2.0)$ and $6-9.5 \%$ for $(\mathrm{A}=5.0)$ for variable modified Rayleigh number.

Al-Timemy [6] shows numerically the effect of an adiabatic internal flow partition on natural convection heat transfer in two-dimensional enclosure filled porous media using Darcy flow. The boundary conditions for the cavity are differently heated from sides with insulated horizontal walls. He used more than one partition fixed vertically on the horizontal insulated lower wall. Results presented reduction in heat transfer with increase in the partition and the change in the length of partition is more effect on heat transfer from change in position. 
In this paper, a numerical solution will be used to study the non-Darcian flow effect on natural convection heat transfer in a two-dimensional rectangular enclosure, which is filled with saturated porous medium heated from below with partition. The partition had a variable width and located at different places from the insulated walls.

\section{Physical Model:}

For the problem geometry shown in figure (1), the enclosure filled with saturated porous medium with natural convection inside the solid matrix. The enclosure is subjected to the indicated boundary conditions, the vertical walls are isothermal at different temperature, while the horizontal walls are insulated.

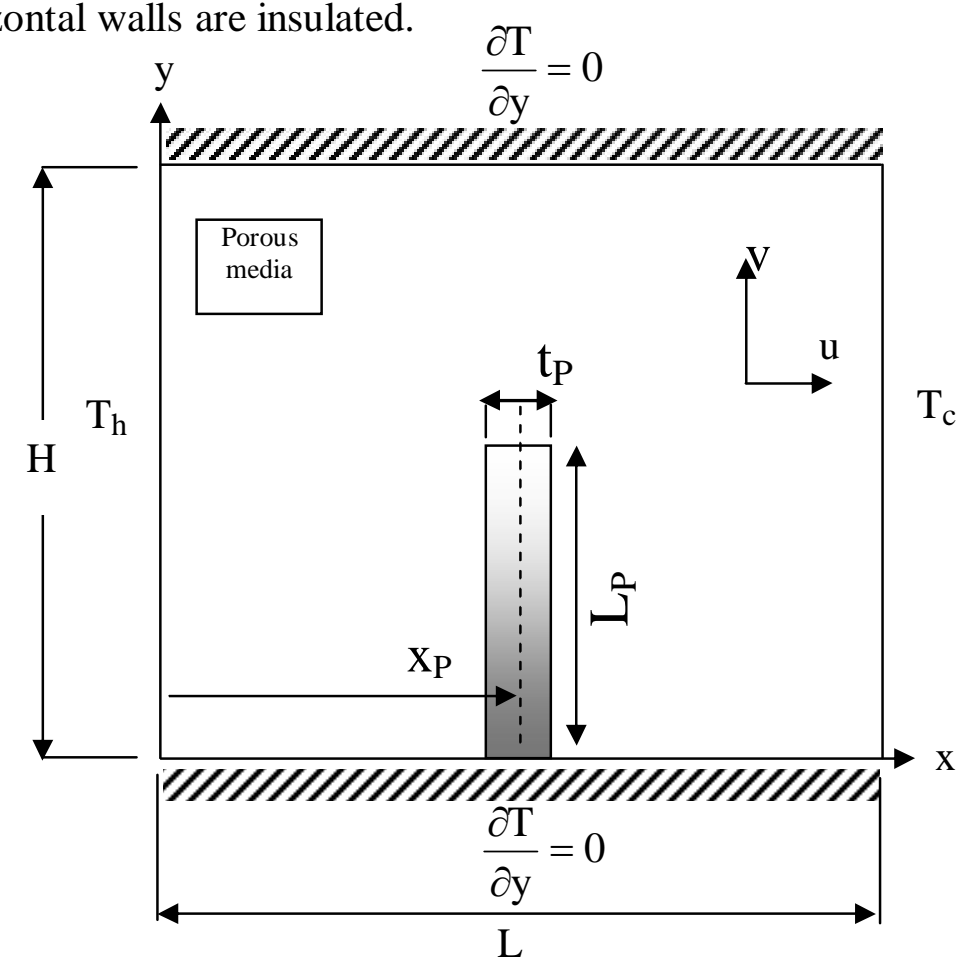

shows the schematic diagram for the problem Figure (1)

To simplify the analysis, the following assumptions are made:

1-the fluid is incompressible, steady and laminar

2-A continuum saturated porous medium

3-A two-dimensional distribution of fluid flow and temperature

4-Homogeneous and isotropic properties of the fluid and solid matrix

5-Thermal equilibrium between the fluid and solid matrix

6-Impermeable boundary surfaces

7-Constant porosity medium

8 -Viscous term is neglected

Equations which govern the heat transfer and fluid flow in a saturated porous media are:

1 -The continuity equation for the steady form and incompressible flow is given by:

$\frac{\partial \mathrm{u}}{\partial \mathrm{x}}+\frac{\partial \mathrm{v}}{\partial \mathrm{y}}=0$

2-The energy equation which depending on the considered assumption, the steady flow and constant property of fluid with no heat generation energy equation is:

$\mathrm{u} \frac{\partial \mathrm{T}}{\partial \mathrm{x}}+\mathrm{v} \frac{\partial \mathrm{T}}{\partial \mathrm{y}}=\alpha\left[\frac{\partial^{2} \mathrm{~T}}{\partial \mathrm{x}^{2}}+\frac{\partial^{2} \mathrm{~T}}{\partial \mathrm{y}^{2}}\right]$ 


\section{Hussain : Effect Of Non-Darcian Flow On Natural Convection Inside Rectangular}

Where $\alpha$ is effective thermal diffusivity of the medium of the medium

3-The momentum equation which depending on the Brinkman-Forchheimer equation which is a modification to Darcy equation of motion is porous media after including the boundary layer and inertia effects. The momentum equation in $\mathrm{x}$-direction is:

$\frac{\mu}{\mathrm{K}} \mathrm{u}+\frac{\rho b}{\mathrm{~K}}|\mathrm{~V}| \cdot \mathrm{u}=-\frac{\partial \mathrm{p}}{\partial \mathrm{x}}$

And in y-direction is:

$\frac{\mu}{\mathrm{K}} \mathrm{v}+\frac{\rho \mathrm{b}}{\mathrm{K}}|\mathrm{V}| \cdot \mathrm{v}=-\frac{\partial \mathrm{p}}{\partial \mathrm{y}}+\rho \mathrm{g}$

Using the Boussinesq approximation in the body force term $(\rho g)$ of equation (4) and to eliminate the pressure term by cross-differentiating equations (3) and (4) and subtract, then:

$\frac{\mu}{K}\left[\frac{\partial u}{\partial y}-\frac{\partial v}{\partial x}\right]+\frac{\rho b}{K}\left[\frac{\partial}{\partial y}(|V| \cdot u)-\frac{\partial}{\partial x}(|V| \cdot v)\right]=+\rho_{o} g \beta \frac{\partial T}{\partial x}$

The above equations to dimensionless formulation, the momentum equation and the energy equation are converted respectively yields to:

$\frac{\partial^{2} \hat{\psi}}{\partial \hat{\mathbf{y}}^{2}}+\frac{\partial^{2} \hat{\psi}}{\partial \hat{\mathbf{x}}^{2}}=\frac{\mathrm{F}_{\mathrm{S}}}{\operatorname{Pr}^{*}}\left[\frac{\partial}{\partial \hat{\mathrm{y}}}(|\hat{\mathrm{V}}| \cdot \hat{\mathrm{u}})-\frac{\partial}{\partial \hat{\mathbf{x}}}(|\hat{\mathrm{V}}| \cdot \hat{\mathrm{v}})\right]+\operatorname{Ra}^{*} \frac{\partial \theta}{\partial \hat{\mathbf{x}}}$

and

$\frac{\partial \hat{\psi}}{\partial \hat{x}} * \frac{\partial \theta}{\partial \hat{y}}-\frac{\partial \hat{\psi}}{\partial \hat{y}} * \frac{\partial \theta}{\partial \hat{x}}=\frac{\partial^{2} \theta}{\partial \hat{x}^{2}}+\frac{\partial^{2} \theta}{\partial \hat{y}^{2}}$

Where:

$\widehat{\mathrm{x}}=\frac{\mathrm{x}}{\mathrm{L}}, \widehat{\mathrm{y}}=\frac{\mathrm{y}}{\mathrm{L}}$ and $\mathrm{A}=\frac{\mathrm{H}}{\mathrm{L}}$

$\theta=\frac{\mathrm{T}-\mathrm{T}_{\mathrm{c}}}{\mathrm{T}_{\mathrm{h}}-\mathrm{T}_{\mathrm{c}}}=\frac{\mathrm{T}-\mathrm{T}_{\mathrm{c}}}{\Delta \mathrm{T}}, \mathrm{u}=-\frac{\partial \psi}{\partial \mathrm{y}}, \mathrm{v}=\frac{\partial \psi}{\partial \mathrm{x}}$

$\widehat{\psi}=\frac{\psi}{\alpha},|\mathrm{V}|=\sqrt{\mathrm{u}^{2}+\mathrm{v}^{2}}$

\section{Numerical solution}

There are many methods to construct a computer compatible model of a differential equation. The one chosen is the truncated Taylor series, which is effective and simple in formulation with numerical accuracy in results.

For any unknown variable points $\phi(x, y)$, the continuous first and second order derivatives at grid point $(\mathrm{I}, \mathrm{j})$, which are $(\partial \phi / \partial \mathrm{x})_{\mathrm{i}, \mathrm{j}}$ and $\left(\partial^{2} \phi / \partial \mathrm{x}^{2}\right)_{\mathrm{i}, \mathrm{j}}$ respectively may be expressed in three ways:

The forward difference approximation for the one side three points, the first and second derivatives are:

$$
\begin{aligned}
& \left(\frac{\partial \phi}{\partial \mathrm{x}}\right)_{\mathrm{i}, \mathrm{j}}=\frac{-3 \phi_{\mathrm{i}, \mathrm{j}}+4 \phi_{\mathrm{i}+1, \mathrm{j}}-\phi_{\mathrm{i}+2, \mathrm{j}}}{2 \Delta \mathrm{x}}+\mathrm{O}(\Delta \mathrm{x})^{2} \\
& \left(\frac{\partial^{2} \phi}{\partial \mathrm{x}^{2}}\right)_{\mathrm{i}, \mathrm{j}}=\frac{\phi_{\mathrm{i}, \mathrm{j}}-2 \phi_{\mathrm{i}+1, \mathrm{j}}+\phi_{\mathrm{i}+2, \mathrm{j}}}{(\Delta \mathrm{x})^{2}}+\mathrm{O}(\Delta \mathrm{x})^{2}
\end{aligned}
$$

The backward difference approximation for the one side three point, the first and second derivatives are: 


$$
\begin{aligned}
& \left(\frac{\partial \phi}{\partial \mathrm{x}}\right)_{\mathrm{i}, \mathrm{j}}=\frac{-3 \phi_{\mathrm{i}, \mathrm{j}}+4 \phi_{\mathrm{i}-1, \mathrm{j}}-\phi_{\mathrm{i}-2, \mathrm{j}}}{2 \Delta \mathrm{x}}+\mathrm{O}(\Delta \mathrm{x})^{2} \\
& \left(\frac{\partial^{2} \phi}{\partial \mathrm{x}^{2}}\right)_{\mathrm{i}, \mathrm{j}}=\frac{\phi_{\mathrm{i}, \mathrm{j}}-2 \phi_{\mathrm{i}-1, \mathrm{j}}+\phi_{\mathrm{i}-2, \mathrm{j}}}{(\Delta \mathrm{x})^{2}}+\mathrm{O}(\Delta \mathrm{x})^{2}
\end{aligned}
$$

Where $\mathrm{O}(\Delta \mathrm{x})^{2}$ is the truncation error of second order.

Both of the forward and backward differences are used to find the derivatives at the boundaries. Similar forms of those derivatives exist for y-direction. The centered difference approximation using three points to calculate the interior points, the first and second derivatives are:

$$
\begin{aligned}
& \mathrm{u} \frac{\partial \mathrm{T}}{\partial \mathrm{x}}+\mathrm{v} \frac{\partial \mathrm{T}}{\partial \mathrm{y}}=\alpha\left[\frac{\partial^{2} \mathrm{~T}}{\partial \mathrm{x}^{2}}+\frac{\partial^{2} \mathrm{~T}}{\partial \mathrm{y}^{2}}\right] \\
& \mathrm{u} \frac{\partial \mathrm{T}}{\partial \mathrm{x}}+\mathrm{v} \frac{\partial \mathrm{T}}{\partial \mathrm{y}}=\alpha\left[\frac{\partial^{2} \mathrm{~T}}{\partial \mathrm{x}^{2}}+\frac{\partial^{2} \mathrm{~T}}{\partial \mathrm{y}^{2}}\right]
\end{aligned}
$$

Using the finite difference forms for the derivatives in the non-dimensional forms of the governing equations to get the momentum equation, it will be in the finite difference as:

$$
\begin{aligned}
& \frac{\hat{\psi}_{i, j+1}-2 \hat{\psi}_{i, j}+\hat{\psi}_{i, j-1}}{(\Delta \hat{y})^{2}}+\frac{\hat{\psi}_{i+1, j}-2 \hat{\psi}_{i, j}+\hat{\psi}_{i-1, j}}{(\Delta \hat{x})^{2}}=\operatorname{Ra}\left(\frac{\theta_{i+1, j}-\theta_{i-1, j}}{2 \Delta \hat{x}}\right) \\
& +\frac{F_{s}}{\operatorname{Pr}}\left[\left\{\frac{|\hat{V}|_{i, j+1} * u_{i, j+1}-|\hat{V}|_{i, j-1} * u_{i, j-1}}{2 \Delta \hat{y}}\right\}-\left\{\frac{|\hat{V}|_{i+1, j} * v_{i+1, j}-|\hat{V}|_{i-1, j} * v_{i-1, j}}{2 \Delta \hat{x}}\right\}\right]
\end{aligned}
$$

\section{Where :}

$u_{i, j}=\frac{\hat{\Psi}_{i, j-1}-\hat{\Psi}_{i, j+1}}{2 \Delta \hat{y}}, v_{i, j}=\frac{\hat{\Psi}_{i+1, j}-\hat{\Psi}_{i-1, j}}{2 \Delta \hat{x}},|\hat{v}|_{i . j}=\left|\sqrt{\left(\hat{u}_{i, j}\right)^{2}+\left(\hat{v}_{i, j}\right)^{2}}\right|$

and the energy equation as:

$$
\begin{aligned}
& -\left(\frac{\hat{\psi}_{i, j+1}-\hat{\psi}_{i, j-1}}{2 \Delta \hat{y}}\right)\left(\frac{\theta_{i+1, j}-\theta_{i-1, j}}{2 \Delta \hat{x}}\right)+\left(\frac{\hat{\psi}_{i+1, j}-\hat{\psi}_{i-1, j}}{2 \Delta \hat{x}}\right)\left(\frac{\theta_{i, j+1}-\theta_{i, j-1}}{2 \Delta \hat{y}}\right) \\
& =\left(\frac{\theta_{i+1, j}-2 \theta_{i, j}+\theta_{i-1, j}}{(\Delta \hat{\mathrm{x}})^{2}}\right)+\left(\frac{\theta_{i, j+1}-2 \theta_{i, j}+\theta_{i, j-1}}{(\Delta \hat{y})^{2}}\right)
\end{aligned}
$$

The boundary conditions for the enclosure and partition can be written as:

For cavity:

At $\hat{\mathrm{x}}=0,0<\mathrm{y}<\mathrm{A}, \theta=1, \hat{\psi}=0, \mathrm{u}=0, \mathrm{v}=0$

At $\hat{\mathrm{x}}=1,0<\mathrm{y}<\mathrm{A}, \theta=0, \hat{\psi}=0, \hat{\mathrm{u}}=0, \hat{\mathrm{v}}=0$

At $\hat{y}=0, A, 0<x<1, \frac{\partial \theta}{\partial \hat{y}}=0, \hat{\psi}=0, \hat{u}=0, \hat{v}=0$ 


\section{Hussain : Effect Of Non-Darcian Flow On Natural Convection Inside Rectangular}

For partition:

At $\hat{x}=\frac{X_{p}-t_{p}}{L}, \hat{x}=\frac{X_{p}+t_{p}}{L}$ where $0<y<\frac{L p}{L}, \frac{\partial \theta}{\partial \hat{x}}=0, \hat{\psi}=0, \hat{u}=0, \hat{v}=0$

At $\hat{y}=\frac{L p}{L} \frac{X p-\frac{t_{p}}{2}}{L}<\hat{x}<\frac{X p+\frac{t_{p}}{2}}{L}, \frac{\partial \theta}{\partial \hat{y}}=0, \hat{\psi}=0, \hat{u}=0, \hat{v}=0$

$\mathrm{Q}_{\mathrm{total}}=\int_{0}^{\mathrm{A}}\left(\frac{\partial \theta}{\partial \mathrm{x}}\right)_{\mathrm{x}=0, \mathrm{~L}} \mathrm{dy}$

$\mathrm{Nu}=\frac{1}{\mathrm{~A}} \int_{0}^{\mathrm{A}}\left(\frac{\partial \theta}{\partial \mathrm{x}}\right)_{\mathrm{x}=0, \mathrm{~L}} \mathrm{dy}$

\section{Discussion:}

Before presenting the results for the various parameters, it's worthwhile discussing the different flow regime encountered in free convection in a porous medium enclosure. Convection heat transfer in a porous cavity is usually classified as belonging to the conduction, the asymptotic, and the boundary layer flow regimes [7] depending on the temperature difference between the two isothermal vertical walls denoted by the increase in Rayleigh number. The boundary layer regime will appear at a certain range of modified Rayleigh number [8].

The range considered in this work falls within the merged layer regime where the horizontal layer apparently fills the cavity and a thin vertical boundary layer will appear. In the convection heat transfer both of the heat and fluid flow fields are coupled and interdependent. However, it is convenient to present and discuss the fluid flow field first, and then proceed with the heat flow field. The fluid flow is presented as stream lines which indicate the flow direction and magnitude. After that, the lines of constant temperature (isotherms) are presented, to quantify the heat flow field. Finally, the temperature field discussed completing the field result presentation.

The present study was carried out for the condition of the partition being attached at the lower wall with different position and different length for aspect ratio $(A=1.0)$. The streamlines in figures ( 2 to 5 ) show a circulation of fluid to be clockwise around a point of maximum stream function and the boundary layer regime being near the isothermal wall. The partition deflects stream line vertically causing a disturbance in the flow pattern and displace the point of $\psi_{\max }$ upward towards the hot wall and the flow turns around the partition, the core stratification at the a upper part of enclosure will note be affect hardly.

The elongation of the partition makes the flow to be increased near the hot wall and decreased at the lower part of the cold wall until a stagnation of flow occurs as the partition be longer due to separation of flow from the cold wall. Also, the isotherms deflect upward and the temperature gradient decreased with partition elongation and the boundary layer thickness decrease near the hot and the cold walls and the heat transfer become lower as less area of heat transfer will be available when the partition elongated and the stagnation regime increased near the cold wall as shown in figure(2) . 

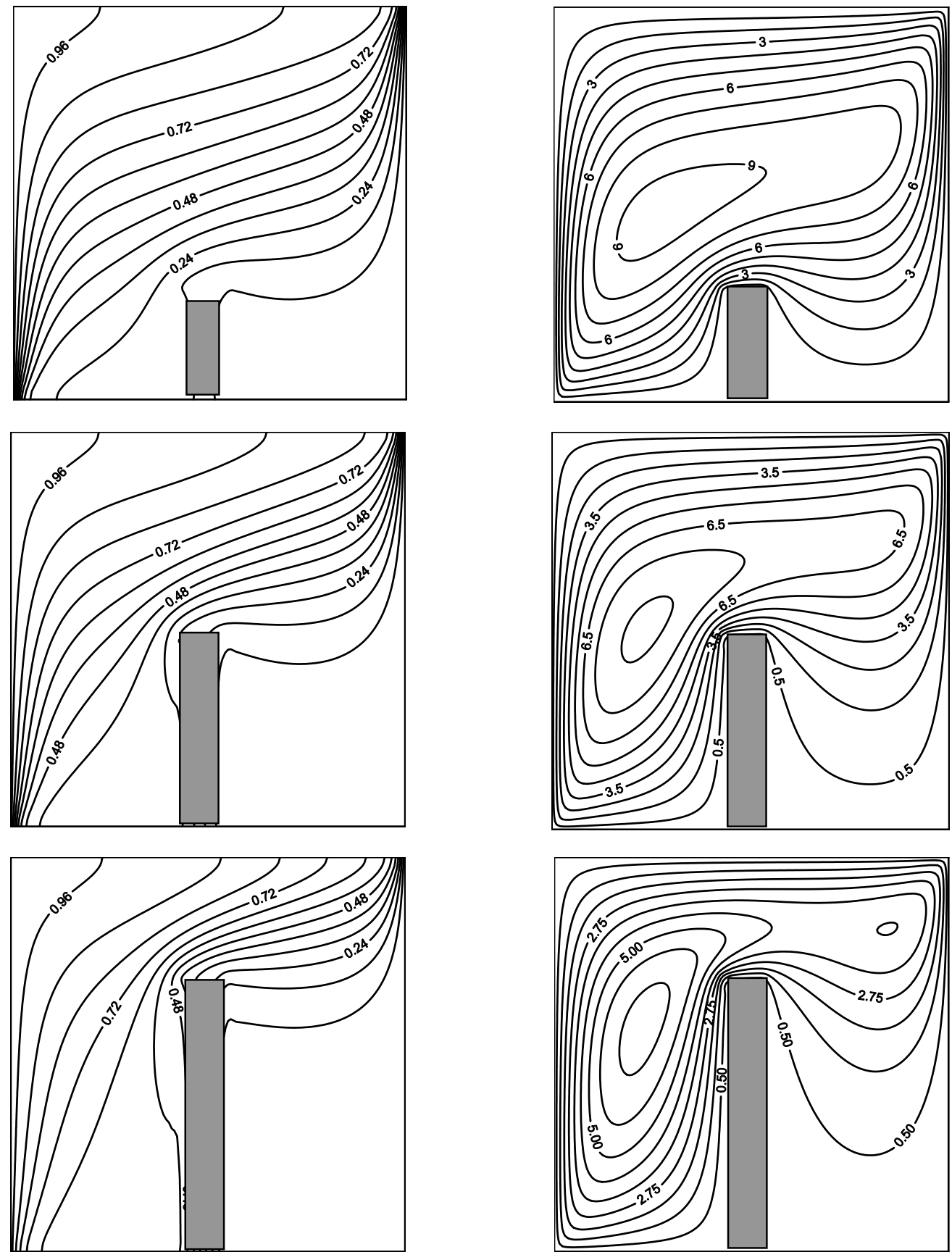

Fig ( 2 ) Isotherms lines \& Stream functions for $\mathrm{Xp}=0.5, \mathrm{Ra}=300, \mathrm{Fs} / \mathrm{Pr}=0.0001$ (a) $\mathrm{Lp}=0.3$, (b) $\mathrm{Lp}=0.5$, (c) $\mathrm{Lp}=0.7$

When the partition location changes towards the cold wall, the value of maximum stream function increases and the rotation core becomes lower to indicate an increase in the fluid velocity. The stagnation regime at the lower part of the cold wall grew causing a decrease in the heat transfer rate at that part. A less isotherm deflection as the partition moved toward cold wall and a more effects for the thermal boundary layer near the hot and cold walls and the stratification increased above the partition regime as shown in figure (3). 



Fig ( 3 ) Isotherms lines \& Stream functions for $\mathrm{Lp}=0.7, \mathrm{Ra}=800, \mathrm{Fs} / \mathrm{Pr}=0.01$ (a) $\mathrm{Xp}=0.3$, (b) $\mathrm{Xp}=0.5$, (c) $\mathrm{Xp}=0.7$

The increase the inertia effect (Fs/Pr) causes a reduction in $\psi_{\max }$ and flow circulation, also the boundary layer thickness is reduced near the vertical walls causing a reduction in the natural convection. Figure (4) shows the inertia effect represented by (Fs/Pr) with fixing the other parameter. The isotherm at the lower part near partition in much affected and separated from hot wall due to inertia effect, also the temperature gradient becomes lower. 


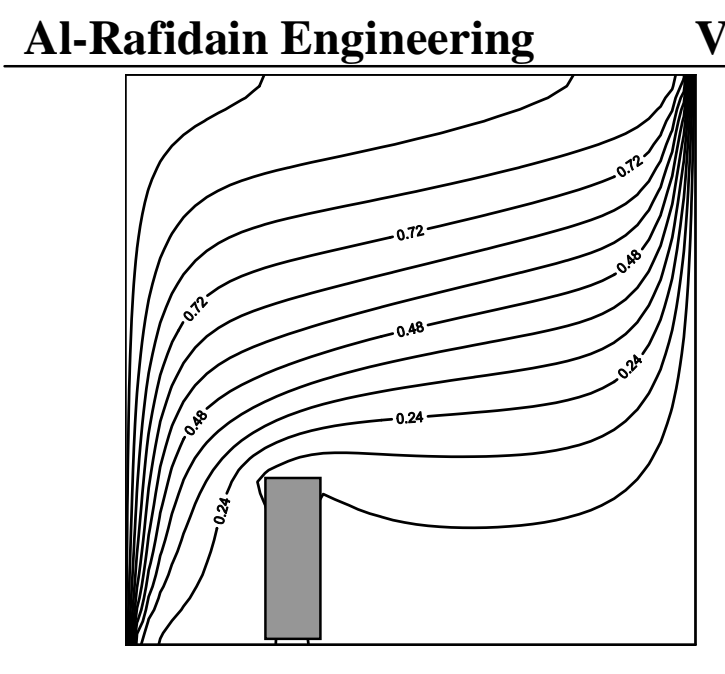

Vol.17 No.3

June 2009
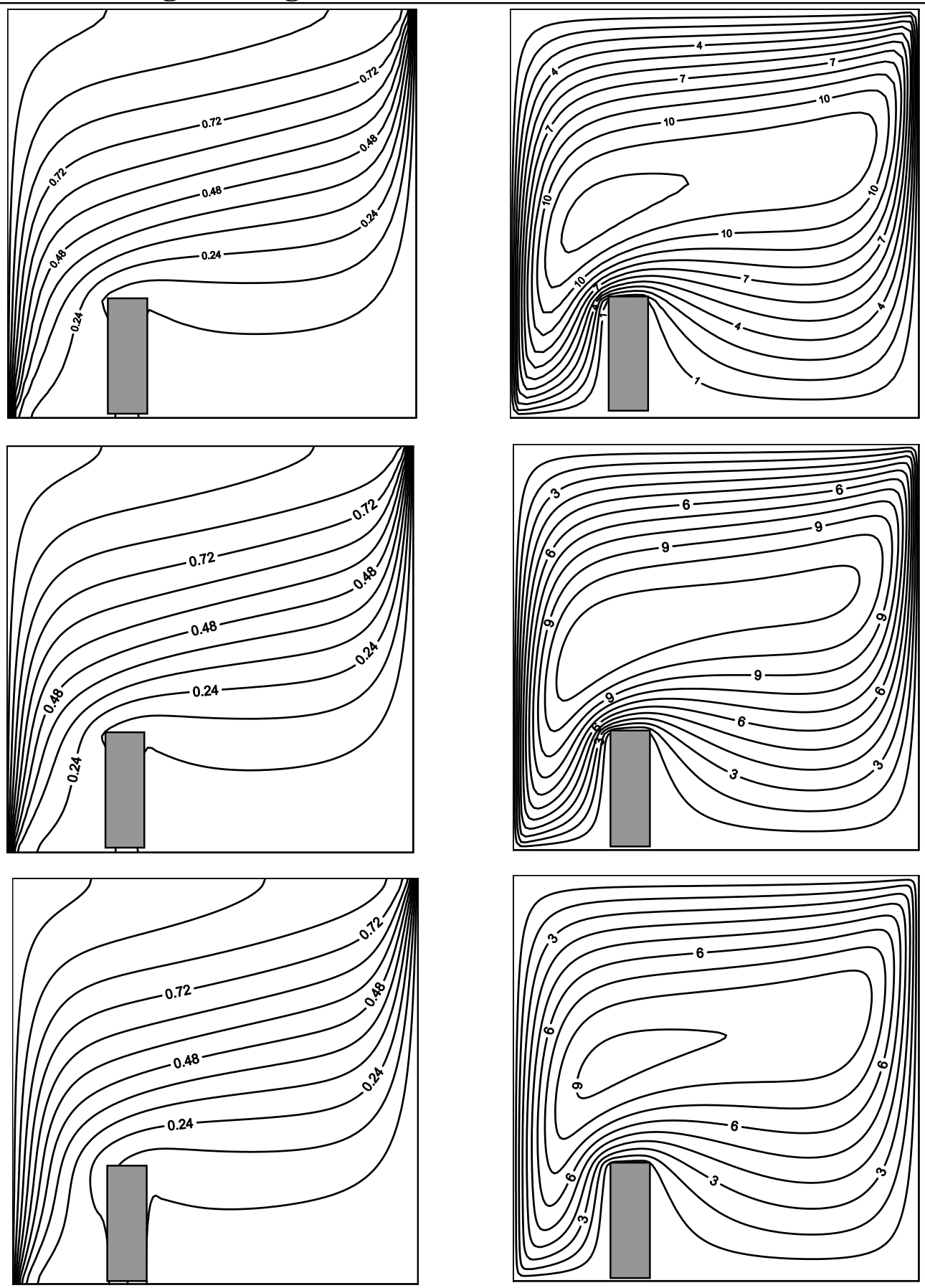

Fig ( 4 ) Isotherms lines \& Stream functions for

$\mathrm{Lp}=0.3, \mathrm{Xp}=0.3, \mathrm{Ra}=500 \mathrm{Fs} / \mathrm{Pr}=0.0001$, (b) Fs $/ \mathrm{Pr}=0.001$, (c) $\mathrm{Fs} / \mathrm{Pr}=0.01$

Figure (5) indicates the effect of increasing of Rayliegh number causing an increase in $\psi_{\max }$, the increased flow velocity causes an increase in the natural heat transfer, also the boundary layer thickness near the hot and cold walls increased due to the increase in the bouncy force. This affects the isotherm to be clear near the lower part of the hot wall and the temperature gradient will be increased causing a bigger difference in the fluid density and increase the bouncy force and more heat will be transferred to the fluid and carried to the cold wall causing a rise in the heat transfer rate. 


\section{Hussain : Effect Of Non-Darcian Flow On Natural Convection Inside Rectangular}
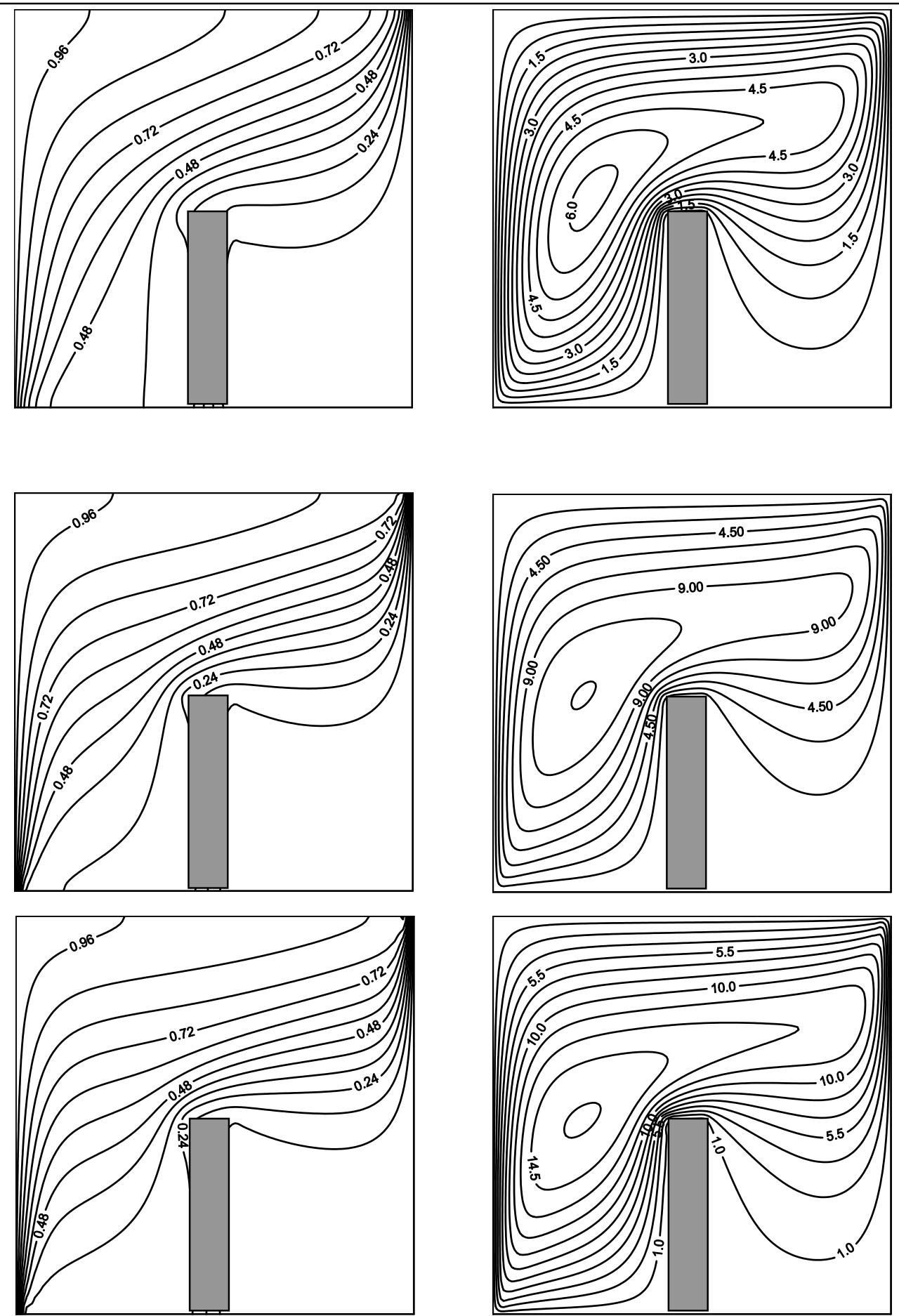

Fig ( 5 ) Isotherms lines \& Stream functions for

$\mathrm{Lp}=0.5, \mathrm{Xp}=0.5, \mathrm{Fs} / \mathrm{Pr}=0.001$ (a) $\mathrm{Ra}=200$, (b) $\mathrm{Ra}=500$, (c) $\mathrm{Ra}=800$

The Nusselt number will be considered as an indication to the heat transfer rate for different parameter effects. The Rayliegh number variation demonstrates a significant effect on Nusselt number. Those effects are presented in figure (6) which shows the variation of Nusselt number with Rayliegh number, for different effect of parameter studied. All figures indicate that Nusselt number increased when Rayliegh number increased for all studied parameter. The increasing in Rayliegh number increases the fluid flow and so the heat transfer rate. The figure show the effect of different partition location $\left(\mathrm{X}_{\mathrm{P}}=0.3,0.5\right.$ and 0.7$)$ and 
partition length $\left(\mathrm{L}_{\mathrm{P}}=0.3,0.5\right.$ and 0.7$)$ at different inertia effect $(\mathrm{Fs} / \mathrm{Pr}=0.01,0.001$ and 0.0001$)$. Figure (6) shows for all (Ra) and for each partition length, when the partition is being closed to cold wall $\left(\mathrm{X}_{\mathrm{p}}=0.7\right)$, the Nusselt number increased with lower gradient, this effect is due to more area for fluid to be flow in front of hot wall and more heat is transferred to the fluid.

For the same partition locations $\left(\mathrm{X}_{\mathrm{p}}=0.3,0.5,0.7\right)$ as the length of partition increase the Nusselt number decrease, this variation being with Rayliegh numbers less than (400) and become more significant at higher Rayliegh, because of the effect of inertia that be more significant at high Rayliegh number. More elongation of the partition caused a reduction of Nusselt number for all Rayliegh and Fs/Pr that is due to reduction of area of heat transfer and increased of the stagnation behind the partition near the cold wall.
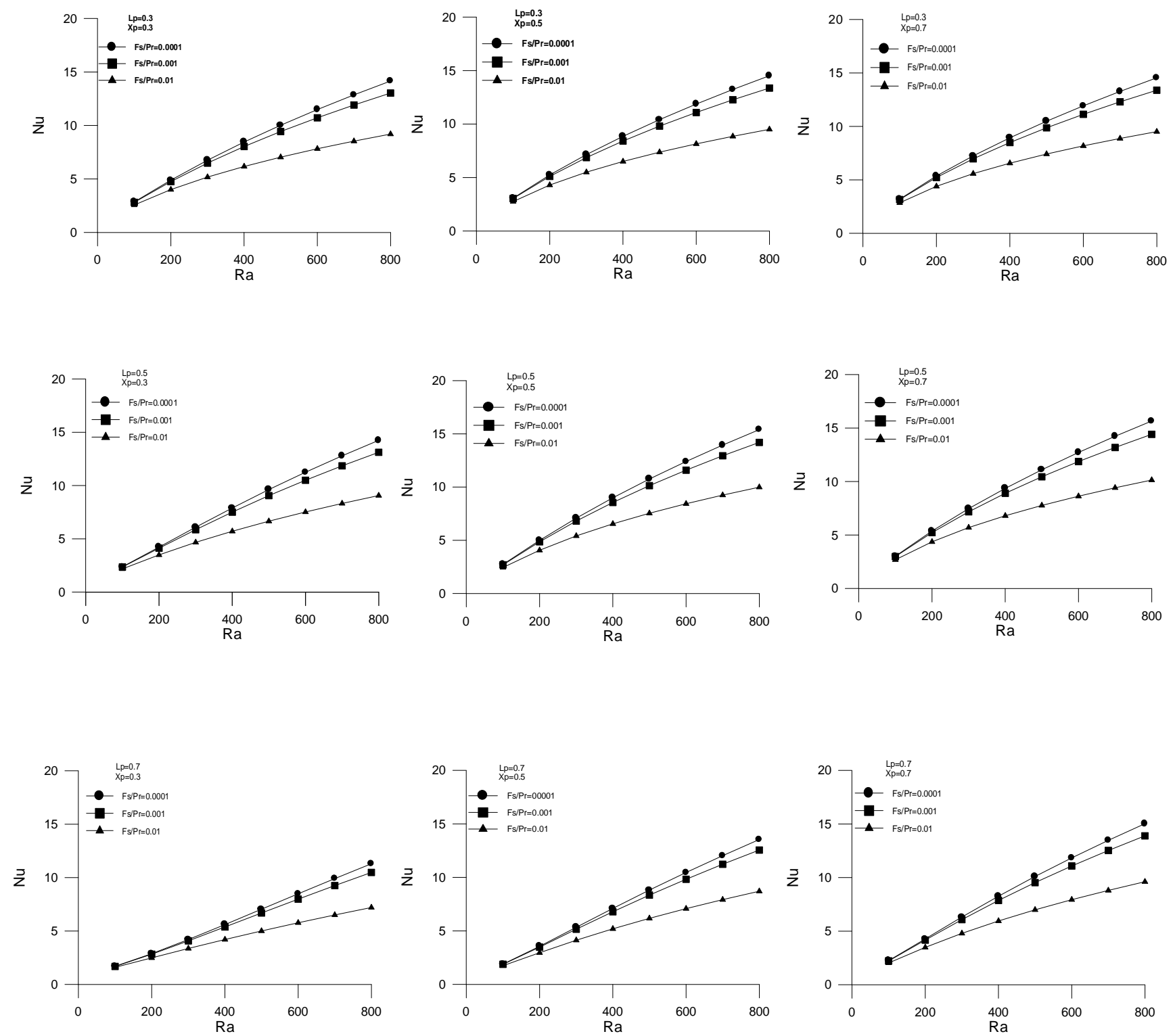

Fig (6) Variation of Nusselt number with Rayliegh number for different partition length with various different location and internal effect 


\section{Hussain : Effect Of Non-Darcian Flow On Natural Convection Inside Rectangular}

\section{Conclusions:}

The results obtained by this study indicate many conclusions:

1- The partition reduces the heat transfer rate and has an insulating effect.

2- The increase of partition length reduces the heat transfer rate.

3- When the partition location moving toward the cold wall, the heat transfer increases.

4- The partition distorts the streamlines with disturbance in the flow pattern and change the isotherms shapes.

5- The increase in the inertia parameter $(\mathrm{Fs} / \mathrm{Pr})$ at high $(\mathrm{Ra})$ enhances the boundary layer thickness, reduces the velocity which causes a reduce in heat transfer.

\section{References:}

1-Chen B.K., Ivey C.M., \& Barry J.M., "Natural Convection in Enclosed Porous Media with Rectangular Boundaries" Journal of Heat Transfer, Feb. 1970

2-Tong T.W., \& Subramanian E., "A Boundary Layer Analysis for Natural Convection in vertical Porous Enclosure-Use of the Brinkman-Extend Darcy Model", Int. J. Heat Mass Transfer, Vol.28,pp.563-571,1985.

3-Prasad V., and Tuntome A.," Inertia Effect on Natural convection in a Vertical Porous Cavity", Numer. Heat Transfer, Vol.11, pp.295-320, 1987.

4-Beckermann C., Ramadhyani S., and Viskanta R., "Natural Convection Flow and Heat transfer Between a fluid Layer and Porous Layer Inside a Rectangular Enclosure", J. Heat Transfer, Vol. 109, pp.366-370,1987.

5-Hussein, A.," Effect of Non-Darcian Flow on Natural Convection in Partitioned Enclosure", Ph.D. Thesis, university of Mosul, 2001.

6-Al-Tamemy,M., "Numerical study of Natural Convection in Porous Enclosure with Multi-Obstractions", M.Sc. Thesis, University of Mosul, 2003

7-Prasad V., and Kulaki F. A.," Convection Heat Transfer in a Rectangular Porous Cavity - Effect of Aspect Ratio on Flow Structure and Heat Transfer", Journal of Heat Transfer, ASME, Vol.106, pp.158-165, February 1984.

8-Blythe P. A., Simpkins P. G., and Daniels P.G.," Thermal Convection in a Cavity Filled with a Porous Medium: a Classification of Limiting Behaviors", Int. J. Heat Mass Transfer. Vol.26, No.5, pp.701-708, 1983. 